\title{
Intramedullary fibula strut bone allograft in a periprosthetic humeral shaft fracture with implant loosening after total elbow arthroplasty
}

\author{
Young-Hoon Jo ${ }^{1}$, Seung Gun Lee ${ }^{2}$, Incheol Kook ${ }^{2}$, Bong Gun Lee ${ }^{2}$ \\ ${ }^{1}$ Department of Orthopedic Surgery, Hanyang University Guri Hospital, Guri, Korea \\ ${ }^{2}$ Department of Orthopedic Surgery, Hanyang University College of Medicine, Seoul, Korea
}

\begin{abstract}
Periprosthetic fracture after total elbow replacement surgery is a difficult complication to manage, especially when it comes together with implant loosening. If stem revision and internal fixation of the periprosthetic fracture are performed simultaneously, this would be a very challenging procedure. Most of total elbow replacement implants are cemented type. Cement usage at periprosthetic fracture site may interfere healing of fractured site. Authors underwent internal fixation with use of locking plate and cerclage wire for periprosthetic fracture, allogenous fibular strut bone inserted into the humerus intramedullary canal allowing the fractured site to be more stable without cement usage. At 10-month follow-up, the complete union and good clinical outcome was achieved. We present a novel technique for treating periprosthetic fracture with implant loosening after total elbow replacement surgery, using intramedullary allogenous fibula strut bone graft.
\end{abstract}

Keywords: Elbow; Periprosthetic fracture; Arthroplasty

Total elbow replacement was used first in treatment of rheumatoid arthritis patients in 1970 [1]. Development of implants and better surgical techniques have led to widespread use of this method in various elbow diseases, such as osteoarthritis, posttraumatic arthritis, severe comminuted fracture with bone loss, chronic instability, and tumors [2,3]. However, total elbow replacement can cause various complications, such as aseptic loosening, infection, and periprosthetic fractures, during long-term follow-up. To address these issues, revision surgery has been reported [4].

Periprosthetic fracture is widely recognized as a complication of total hip or knee replacement but may also occur following to- tal elbow replacement [5]; the incidence has been reported to range from $5 \%$ to $29 \%$ [6,7]. Periprosthetic fractures after total elbow replacement are classified according to location, degree of bone loss around the implant, and presence of implant loosening [6]. If the periprosthetic fracture is accompanied by implant loosening, complex techniques such as revision surgery and bone grafting for bone defects are often required in addition to internal fixation. Due to the high rate of complications after surgery with this procedure, it is vital to have an appropriate preoperative surgical plan.

Sanchez-Sotelo et al. [5] reported 11 cases of total elbow replacement with allograft strut bone augmentation and cement

Received: August 3, $2020 \quad$ Revised: August 18, 2020 Accepted: August 18, 2020

Correspondence to: Bong Gun Lee

Department of Orthopedic Surgery, Hanyang University College of Medicine, 222-1 Wangsimni-ro, Seongdong-gu, Seoul 04763, Korea

Tel: +82-2-2290-8485, Fax: +82-2-2290-3774, E-mail: bglee@hanyang.ac.kr, ORCID: https://orcid.org/0000-0002-4003-5529

Financial support: None.

Conflict of interest: None.

Copyright@ 2020 Korean Shoulder and Elbow Society. All Rights Reserved.

This is an Open Access article distributed under the terms of the Creative Commons Attribution Non-Commercial License (http://creativecommons.org/licenses/by-nc/4.0/) which permits unrestricted non-commercial use, distribution, and reproduction in any medium, provided the original work is properly cited. 
fixation that required revision for periprosthetic fracture following total elbow replacement caused by implant loosening. However, six of these 11 cases had poor prognosis with one or more complications, and technical difficulties were reported. If internal fixation and bone graft are performed after fixing the implant with cement, circulation at the fracture site may be compromised by the cement, which will likely delay bone union. In addition, it is also difficult to perform simultaneous internal fixation and bone grafting.

This report introduces a novel surgical technique using an allogenous fibula strut bone graft inserted into the intramedullary canal to improve stability without cement to treat periprosthetic fracture around the humeral stem after total elbow arthroplasty.

\section{TECHNIQUE}

The patient was a 65-year-old woman who had been diagnosed with rheumatoid arthritis 10 years prior. She had recently undergone total elbow replacement surgery using a Coonrad-Morrey (Zimmer, Warsaw, IN, USA) implant at another hospital after sustaining an injury while lifting a heavy object. She had undergone regular bone mineral density testing once a year and did not need medication for osteopenia. Upon radiographic examination, a periprosthetic fracture around the humeral stem corresponding to Mayo classification type H-II was identified, and implant loosening and osteolysis were also observed (Fig. 1).

The authors performed revision of total elbow replacement using the Coonrad-Morrey implant (Zimmer). The previous operative skin incision was used along with a triceps-preserving approach. The incision was deepened to the subcutaneous tissue, and then the triceps fascia was undermined to identify the medial and lateral edges of the triceps muscle. On the medial side, the ulnar nerve was identified and confirmed to be located anteriorly. A longitudinal incision was made on the exposed triceps tendon on the radial side of the midline; care was taken to avoid the tip of the olecranon, and then the joint was exposed. To obtain an adequate view during the approach, the authors elevated the flexor carpi ulnaris and part of the pronator muscle group subperiosteally and released the triceps partially. Loosening of the humeral stem was identified, and osteolysis and metallosis were present. Debridement was carried out with complete removal of all soft tissue around the humeral stem. The ulnar stem was stable with no sign of loosening, so ulnar replacement was not required. After removal of a link between a polyethylene insert and the humeral stem, thorough cement removal and irrigation of the intramedullary canal was performed to avoid influencing the revision surgery. Because the cortical bone around the fracture site was thin and the medullary canal was wide, we opted to perform revision total elbow replacement with a novel technique using an intramedullary fibula bone graft.

For the revision procedure, we used allogenous fibula strut bone (Community Tissue Services, Kettering, OH, USA). After determining the joint line and optimum size of the humeral stem, the diameter and length of the allogenous fibula strut bone were estimated according to the diameter and size of the bone defect and humeral stem. To resolve the difference in diameter between the humeral bone defect lesion and the allogenous fibula strut bone, the latter was trimmed using a high-speed burr to fit the outer diameter to better enter the humeral bone defect area. In addition, the inner diameter of the allogenous fibula
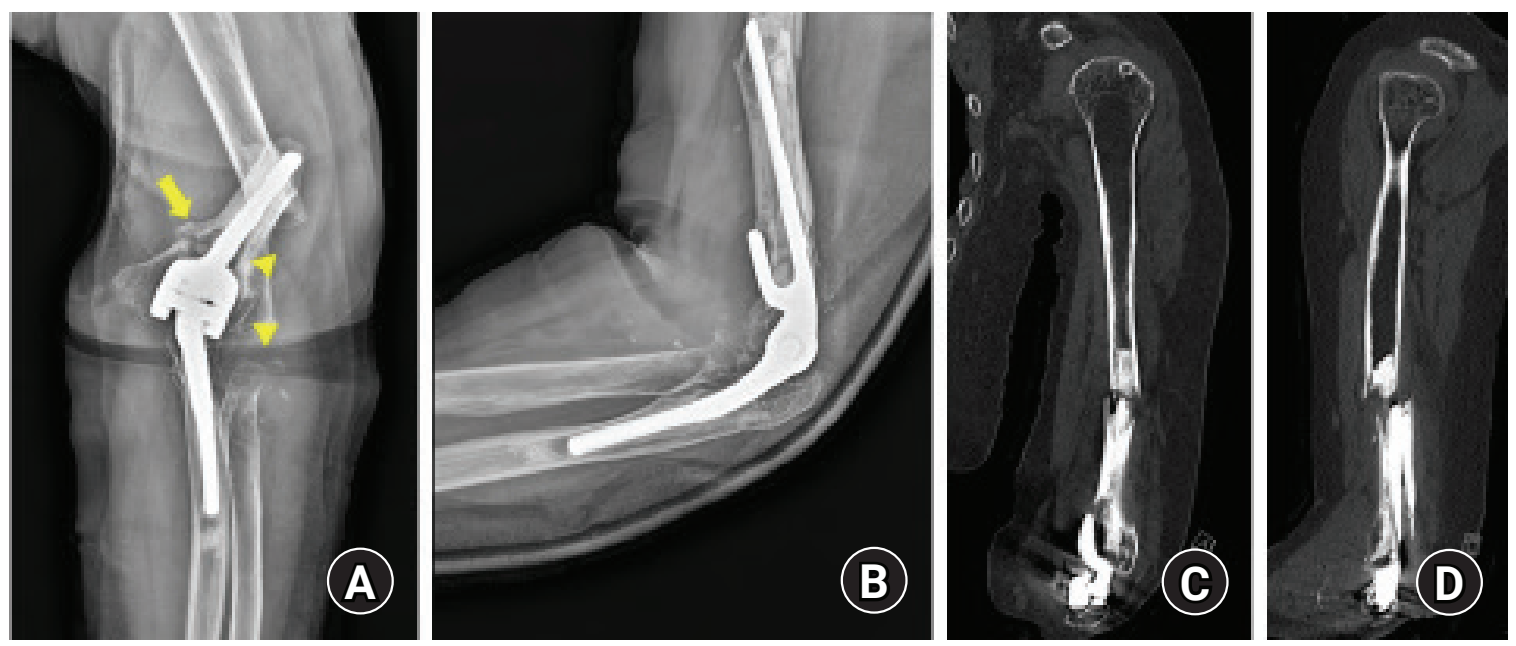

Fig. 1. Elbow anteroposterior (A) and lateral (B) radiographs, coronal (C) and sagittal (D) view of computed tomography images of total elbow arthroplasty with periprosthetic fracture. Previous fracture line are marked with arrow (A), and implant loosening areas are marked with arrowheads $(\mathrm{A})$. 
strut bone was enlarged using a burr to allow the humeral stem to pass (Fig. 2A). Next, it was necessary to confirm whether the allogenous fibula strut bone was located in the fracture site so that the trial stem could pass into the allogenous fibula strut bone before cementation. After closing the proximal portion of the distal fragment from the fracture site using a gauge, we inserted the cement compactly from the distal portion and placed the humeral stem from the distal end. At this time, the cement at the fracture site was removed, and the allogenous fibula strut bone, which contained the humeral stem, was passed through the fracture site. The proximal part of the humeral stem was not performed infusion of bone cement which would inhibit bone union at the fracture site. The fracture site was additionally fixed using a locking plate, eight locking screws, and three cerclage wires (Fig. 2B). After confirming the appropriateness of fixation and bone graft using simple radiographs (Fig. 2C, D), the surgery was complete.

Postoperatively, the elbow was positioned in a long arm splint at a $90^{\circ}$ flexion position for 6 weeks. Two weeks after surgery, the previous long arm splint was replaced with a removable splint, and passive elbow joint motion was performed. Active elbow joint motion was allowed beginning at six weeks after surgery. No other physical therapy or additional fixation was performed. At three months postoperatively, the range of motion (ROM) of the affected elbow was confirmed at $0^{\circ}-110^{\circ}$, and no other complications were observed. At 10 months after surgery, bone union was confirmed on radiographs (Fig. 3A, B), and a satisfactory clinical outcome was obtained with an ROM from $0^{\circ}$ to $150^{\circ}$ of the affected elbow without complications (Fig. 3C, D).
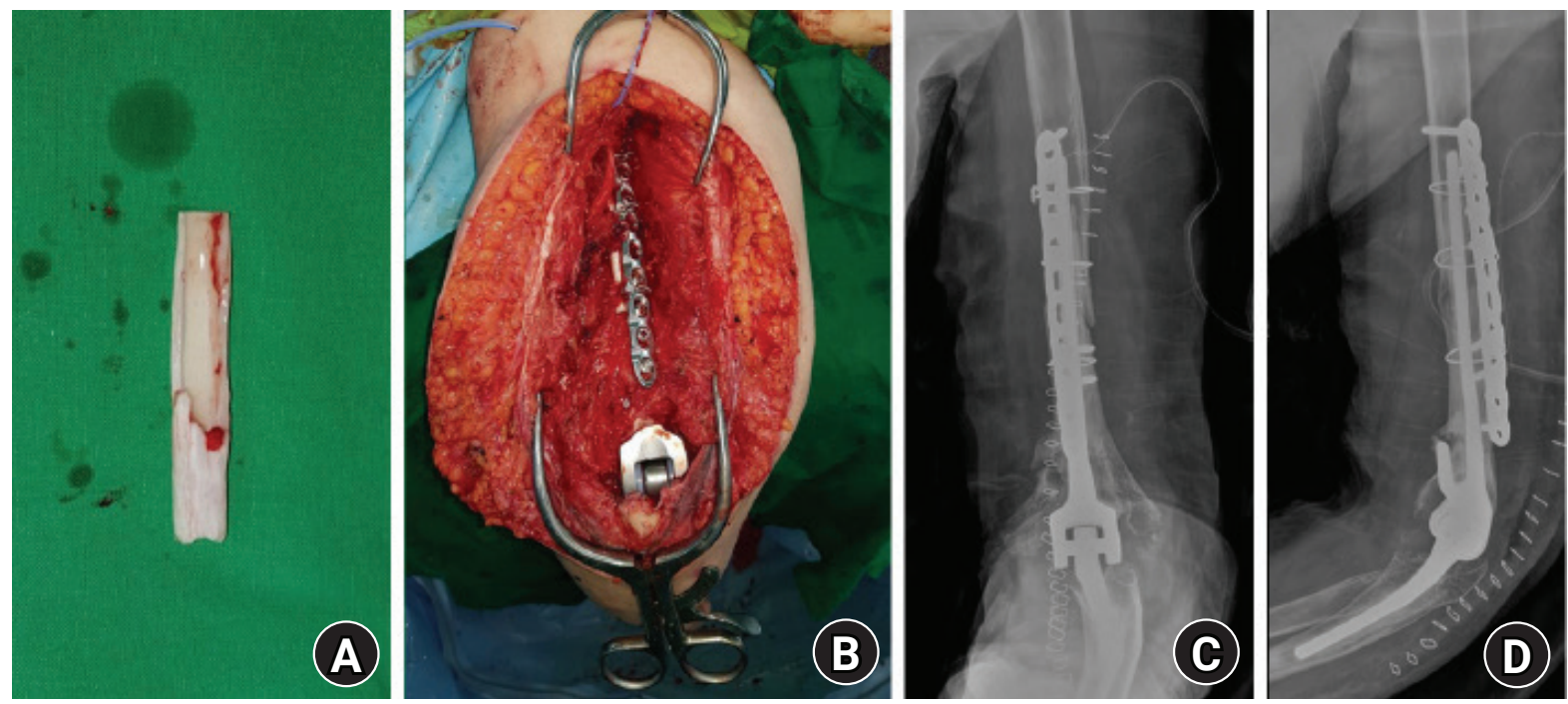

Fig. 2. Allograft fibula strut bone that was manipulated with a high-speed burr (A), and clinical photograph of the operative field after fixation with a locking plate and cerclage wire (B). Anteroposterior (C) and lateral (D) radiographs after operation.
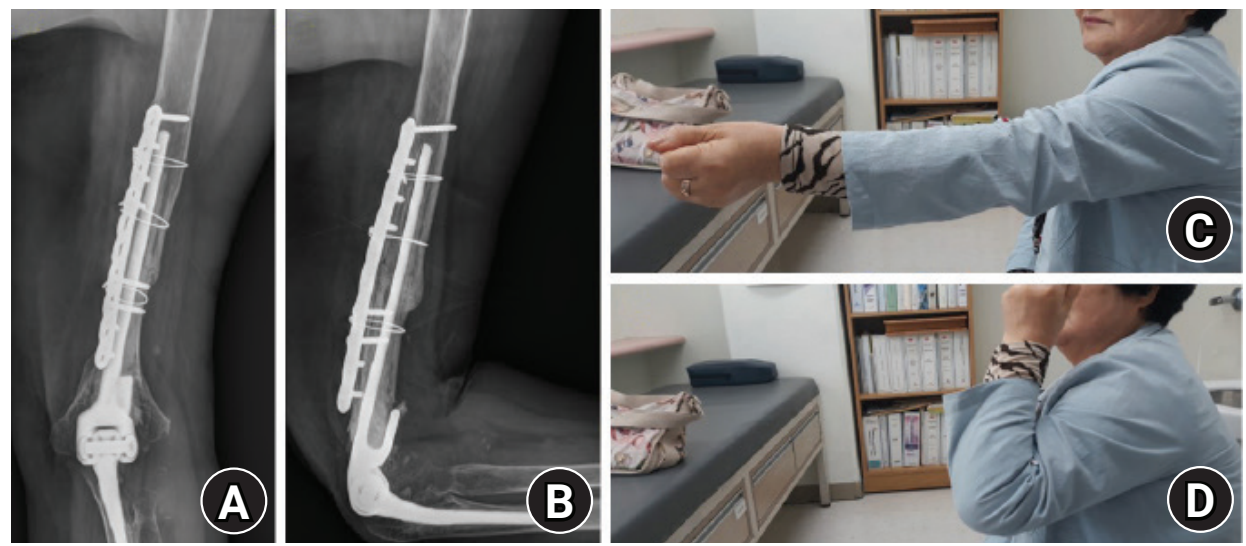

Fig. 3. Anteroposterior (A) and lateral (B) radiographs at postoperative 10 months. Postoperatively, fibula strut-graft incorporation and union were observed with satisfactory clinical outcome and range of motion $0^{\circ}$ to $15^{\circ}(\mathrm{C}, \mathrm{D})$. 


\section{DISCUSSION}

Periprosthetic fracture following total elbow replacement surgery is graded using the Mayo classification system according to fracture site, fixation status of the implant, and bone stock around the implant [5,6]. Different operative techniques may be required for different stages [5]. Treatment strategy can be chosen based on type of humeral shaft fracture [7]. Non-displaced periprosthetic fracture without loosening of the implant is treated with conservative treatment, including long arm splint fixation [6], whereas fracture with implant loosening should undergo revision surgery with internal fixation. Various internal fixation techniques have been reported, including allogenous strut bone graft [5], locking plate and cerclage wire, and external fixation using the Ilizarov system [7]. To manage humeral bone loss, various methods including humeral shortening, use of a longer humeral stem or bone graft around the anterior flange, use of a tumor prosthesis system, or attachment of a humeral intramedullary nail to the humeral stem followed by autogenous bone graft have been reported [8].

In this case, which was Mayo classification type H-II, implant loosening and osteolysis with periprosthetic fracture prompted revision of the humeral stem, bone graft, and internal fixation. A previously reported technique used an allogenous strut bone graft obtained from outside of the fracture site or an on-lay technique fixed with cerclage wire, which resulted in instability around the fracture site [5]. Even when additional plate fixation is used for stability, allogenous bone graft with cerclage wire may interfere with satisfactory positioning of the plate above the humeral shaft. This technique could cause the fracture site to be bulky and might produce surgical site discomfort. This approach can also give rise to complications such as nerve injury and extensor mechanism rupture [5]. Cement leakage via a fracture gap may also occur, causing cement-related complications and increasing risk of non-union.

In this case, we manipulated the allogenous fibular strut bone into a cylinder shape and inserted it into the humerus shaft in an intramedullary manner to maintain the advantage of allogenous bone graft and to reduce possible complications. This approach improved stability without bone loss or shortening and prevented or reduced cement leakage or cement-related complications because the cement was only injected into the distal area of the humeral stem. This technique also provided good positioning of the plate compared to the on-lay technique as no allogenous bone was present outside the humerus.

The most important and challenging component of this tech- nique was shaping the allogenous fibular strut bone into the appropriate diameter to fit into the humeral intramedullary area and allowing the humeral stem to pass through the inner diameter of the fibular bone graft. Thinning the fibular bone while preserving its shape requires delicate handling and precautions, which may take up a substantial amount of time.

The authors' case showed fracture site union, good elbow joint $\mathrm{ROM}$, and pain relief at 10 months postoperatively, indicating that this novel technique can be considered as a treatment option for periprosthetic fracture with implant loosening after total elbow replacement surgery.

\section{ORCID}

Young-Hoon Jo https://orcid.org/0000-0002-4299-2496 Seung Gun Lee https://orcid.org/0000-0001-7401-7620 Incheol Kook https://orcid.org/0000-0001-8691-5495

Bong Gun Lee https://orcid.org/0000-0002-4003-5529

\section{REFERENCES}

1. Dee R, Sweetnam DR. Total replacement arthroplasty of the elbow joint for rheumatoid arthritis: two cases. Proc R Soc Med 1970;63:653-5.

2. Kozak TK, Adams RA, Morrey BF. Total elbow arthroplasty in primary osteoarthritis of the elbow. J Arthroplasty 1998;13:83742

3. Mighell MA, Dunham RC, Rommel EA, Frankle MA. Primary semi-constrained arthroplasty for chronic fracture-dislocations of the elbow. J Bone Joint Surg Br 2005;87:191-5.

4. DeBernardis DA, Horneff JG, Davis DE, Ramsey ML, Pontes MC, Austin LS. Revision total elbow arthroplasty failure rates: the impact of primary arthroplasty failure etiology on subsequent revisions. J Shoulder Elbow Surg 2020;29:321-8.

5. Sanchez-Sotelo J, O'Driscoll S, Morrey BF. Periprosthetic humeral fractures after total elbow arthroplasty: treatment with implant revision and strut allograft augmentation. J Bone Joint Surg Am 2002;84:1642-50.

6. O'Driscoll SW, Morrey BF. Periprosthetic fractures about the elbow. Orthop Clin North Am 1999;30:319-25.

7. Seo H, Im JH, Lee JY. Treatment of periprosthetic fracture after total elbow replacement arthroplasty. J Korean Fract Soc 2020;33:110-7.

8. Kwak JM, Koh KH, Jeon IH. Total elbow arthroplasty: clinical outcomes, complications, and revision surgery. Clin Orthop Surg 2019;11:369-79. 